\title{
ACTIVITY OF KEFIR GRAINS AGAINST GARDNERELLA VAGINALIS
}

\author{
Salwa S. Afifi ${ }^{1}$, Amany A. El-Sharif', Zeinab H. Helal ${ }^{1 *}$,Eman R. El-Musallamy ${ }^{1,2}$ \\ Address (es): \\ ${ }^{1}$ Department of Microbiology and Immunology Faculty of Pharmacy, Al-azhar University, Cairo, Egypt. \\ ${ }^{2}$ Gynecology Clinic, El-Monib Family Medicine Center, Giza, Egypt. \\ *Corresponding author: zeinabhelal@hotmail.com \\ ABSTRACT
}

https://doi.org/10.36547/be.2020.3.4.69-73

\section{ABSTRACT}

Kefir is a probiotic mixture of bacteria and yeasts. In vitro and animal trials have shown kefir to have antibacterial, antifungal and antiviral activity. The main goal of this study was to determine the antimicrobial susceptibility of Gardnerella vaginalis (G.vaginalis) to kefir. Antimicrobial agents, which were commonly used to treat bacterial vaginosis, and kefir were both tested for antimicrobial activities against G.vaginalis using an agar diffusion method. Kefir was able to inhibit the growth of G.vaginalis. Kefir found to have antimicrobial activity similar to ampicillin, chloramphenicol, ceftriaxone, erythromycin and gentamicin against G.vaginalis isolates. Kefir produced zone of inhibition sizes higher than the zones produced by ciprofloxacin, sulphamethoxazole /trimethoprim and tetracycline. Special attention must be paid to kefir as its activity against G. vaginalis recommends that it deserves assessment in the treatment of infections involving G. vaginalis.

Keywords: Kefir, Gardnerella vaginalis, Agar diffusion method

\section{INTRODUCTION}

Bacterial Vaginosis (BV) is the most common cause of vaginal discharge and characterized by shift of microbiome like Lactobacillus species by opportunistic pathogenic bacteria. BV has been connected to severe gynecological and obstetric problems; it is associated with preterm delivery (Stevens et al., 2004). Moreover, BV increases the chance of spread and acquisition of sexuallytransmitted diseases, for example HSV-2 (Cherpes et al., 2005) and HIV (Cohen et al., 2012). Generally, Gardnerella vaginalis (G. vaginalis) was believed to be the solitary causal agent of this disorder (Catlin, 1992), however its role in the etiology of BV was reduced over the years as the excess of other bacterial species was increasingly associated with the illness (Machado et al., 2013; Alves et al., 2014; Castro et al., 2019). Many studies have been placed G. vaginalis in the highlight. Several researchers reported that $G$. vaginalis had a significantly higher virulence potential than many other BV-associated bacteria, because of its greater tendency to form a biofilm, complex early adhesion and produce cytotoxic effect (Machado et al., 2013; Alves et al., 2014). These findings suggest that $G$. vaginalis may have a principal role in the $\mathrm{BV}$ infection development, flagging the way for several opportunists to colonize the vagina (Machado et al., 2013; Alves et al., 2014; Castro et al., 2019).

Owing to the complex polymicrobial nature of $\mathrm{BV}$, traditional treatment with antibiotics are notorious for their low efficacy and high rates of recurrent infection (Bradshaw et al., 2006; Bostwick et al., 2016).

Inadequate functioning of antibiotics is believed to be due to their failure to complete eradication of $\mathrm{BV}$-associated pathogens; because of emerging antibiotic resistance and to their adverse effect on healthy vaginal microbiota (Bradshaw et al., 2006; Bostwick et al., 2016). For these reasons, alternative therapeutic agents need to be pursued for the treatment of BV.

Probiotic strains are claimed to enhance health through immunomodulatory, metabolic and improve epithelial barrier activities against pathological processes. Probiotics are live microorganisms which, when administered in adequate amounts, confer a health benefit on the host (FAO/WHO, 2001). Many probiotic bacteria produce a broad range of effective antimicrobials, including bacteriocins, lactic acid, and hydrogen peroxide.

Kefir is an example of a probiotic mixture of bacteria and yeasts. Kefir strains have revealed various antifungal and antibacterial activities (Bourrie et al., 2016). The antimicrobial effect of Kefir fermented milk has been tested against a wide range of pathogenic bacterial and fungal species (Bourrie et al., 2016) and found to have antimicrobial activity identical to ampicillin, amoxicillin, azithromycin, ceftriaxone, and ketoconazole against many of these species (Bourrie et al., 2016; Rodrigues et al., 2005).

To our knowledge, there are no reports to date that have investigated the antimicrobial effect of kefir against G. vaginalis. This study was designed to isolate the causative agent $G$. vaginalis from bacterial vaginosis patients with their antibiotic sensitivity pattern and to determine the antimicrobial activity of kefir against $G$. vaginalis and compare them with the activity of antibiotics.

\section{MATERIALS AND METHODS}

\section{Clinical setting and study population}

Specimens were collected from 360 pregnant and non-pregnant women within the age of 18-45 years. Patients, who were diagnosed with malignancy, AIDS, menstruation, were not included in the study. In addition, patients having history of vaginal douche on the day of sample collection or those who had antimicrobial agents within the last thirty days were not included. This study was conducted in patients who attended El-Monib Family Medicine Center, El-Giza, Egypt, Gynecology Clinic; the specimens were submitted to the Microbiology Laboratory, El-Monib Family Medicine Center, El-Giza, Egypt, for routine culture.

\section{Diagnosis for $\mathrm{BV}$ and isolation of $G$. vaginalis}

Diagnosis of BV was done based on Amsel's clinical criteria; by the presence of at least three of the following criteria: $\mathrm{pH}>4.5$, positive amine test, homogeneous vaginal discharge, and presence of clue cells. In addition, BV was diagnosed based on Nugent criteria, and later bacteria were cultured on agar. Specimens were cultured on blood agar, sabaroud dextrose agar, and for $G$. vaginalis Columbia blood agar base with $G$. vaginalis selective supplement was used. All the isolates were identified by standard biochemical methods.

\section{Antibiotic susceptibility testing}

Since guidelines or consensus were unavailable for antimicrobial susceptibility patterns of $G$. vaginalis, the antimicrobial described for anaerobic bacteria by the CLSI document M100S (CLSI, 2016) were used.

All the isolates of $G$. vaginalis obtained by culture were tested for antimicrobial susceptibility by the single disc diffusion method. G. vaginalis cells suspension was adjusted to $0.5 \mathrm{McFarland}$ standard solutions. The cultures were tested

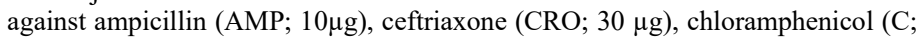
$30 \mu \mathrm{g})$, ciprofloxacin (CIP; $5 \mu \mathrm{g})$, clindamycin (CD; $2 \mu \mathrm{g})$, erythromycin (E; 15 $\mu \mathrm{g})$, gentamycin (GN; $10 \mu \mathrm{g})$, metronidazole (MTZ, $50 \mu \mathrm{g}$ ), sulphamethoxazole/trimethoprim (SXT; $1.25 / 23.7 \mu \mathrm{g}$ ), and tetracycline (TE; 30 $\mu \mathrm{g})$.

\section{Antimicrobial susceptibility of Kefir}

Kefir starter strains were obtained from Yogourmet Company (Canada, Batch \# Q06581). The media for activation of kefir grains was prepared by 100 grams of skim milk to a liter of distilled water, autoclaved at $121^{\circ} \mathrm{C}$ for 15 minutes, and later cooled to $18-20^{\circ} \mathrm{C}$. Twelve gram of Kefir starter per liter was added incubated at $18-20^{\circ} \mathrm{C}$ for 24 hours.

Evaluation of antimicrobial activity of Kefir was done by using the disc diffusion method. Kefir was pipetted on a $5 \mathrm{~mm}$ sterile filter paper disc at the amount of $0.1 \mathrm{ml}$ of $1.2 \mathrm{mg} / \mathrm{ml}$ of kefir as described by Rodrigues et al., (Rodrigues et al., 2005). Disks were applied to the surface of the Human Blood Bilayer Tween agar media earlier swabbed with organism suspension adjusted to $0.5 \mathrm{McFarland}$ 
standard solutions. Inoculated plates were incubated under anaerobic conditions at $37^{\circ} \mathrm{C}$ for 24 hours. Experiments were performed in triplicates, and mean values were calculated.

\section{RESULTS}

A total of 360 women suspected for BV that were between the the ages of 18 to 45 years old were included in this study. Out of 360 patients, based on Amsel criteria, $38(10.6 \%)$ cases were BV positive, and $89.4 \%$ were BV negative. Based on Nugent criteria, 39 patients $(10.8 \%)$ were BV positive, and $89.2 \%$ were BV negative. Microbial culture of vaginal fluid yielded growth of Candida ablicans in 81 patients $(22.5 \%)$, followed by G. vaginalis $(\mathrm{n}=39,10.8 \%)$, then $E$. coli $(\mathrm{n}=21,5.8 \%)$, and Neisseria gonorrheae $(\mathrm{n}=9,2.5 \%)$. Three patients $(0.8 \%)$ had mixed infection of Candida and E. coli. Two hundred and seven $(57.5 \%)$ cases were culture negative

The sensitivity pattern of $G$. vaginalis isolates showed that $94.7 \%$ of isolates were resistant to tetracycline, $74.4 \%$ to sulphamethoxazole/trimethoprim, $64.1 \% \%$ to ciprofloxacin, and $41.0 \%$ to erythromycin. On the other hand, $G$. vaginalis were $100 \%$ sensitive to clindamycin followed by metronidazole $(89.7 \%)$, then gentamycin $(82 \%)$, ampicillin $(76.9 \%)$ and chloramphenicol (74.4\%), table 1 .

Table 1 Antimicrobial susceptibility pattern of G. vaginalis isolated in the study

\begin{tabular}{lccc}
\hline Antimicrobial agents & Sensitive No. (\%) & Intermediate No. (\%) & $\begin{array}{c}\text { Resistance } \\
\text { No. (\%) }\end{array}$ \\
\hline Ampicillin & $30(76.9 \%)$ & $2(5.1)$ & $7(18 \%)$ \\
Cefotriaxone & $35(89.7 \%)$ & $1(2.6)$ & $3(7.7 \%)$ \\
Chloramphenicol & $29(74.4 \%)$ & $5(12.8 \%)$ & $5(12.8 \%)$ \\
Ciprofloxacin & $14(35.9 \%)$ & 0 & $25(64.1 \%)$ \\
Clindamycin & $39(100 \%)$ & 0 & $0(0.0 \%)$ \\
Erythromycin & $24(61.5 \%)$ & $7(17.9 \%)$ & $8(20.5 \%)$ \\
Gentamycin & $32(82.0 \%)$ & $1(2.6 \%)$ & $6(15.4 \%)$ \\
Metronidazole & $35(89.7 \%)$ & 0 & $4(10.3 \%)$ \\
Tetracyclin & $2(5.3 \%)$ & 0 & $37(94.7 \%)$ \\
Sulfamethoxazole/trimethoprim & $10(25.6 \%)$ & 0 & $29(74.4 \%)$ \\
\hline
\end{tabular}

Table 2 The inhibition zones ( $\mathrm{mm}$ ) of kefir and antimicrobial agents against G. vaginalis isolates

\begin{tabular}{|c|c|c|c|c|c|c|c|c|c|c|c|}
\hline Isolate \# & Kefir & Amp & CRO & $\mathbf{C}$ & CIP & CD & $\mathbf{E}$ & TE & GN & MTZ & SXT \\
\hline 1 & 17 & 19 & 25 & 19 & 23 & 30 & 24 & 0 & 18 & 22 & 12 \\
\hline 2 & 20 & 20 & 27 & 20 & 13 & 27 & 26 & 0 & 19 & 25 & 10 \\
\hline 3 & 18 & 19 & 22 & 19 & 12 & 27 & 23 & 0 & 17 & 24 & 9 \\
\hline 4 & 13 & 13 & 24 & 21 & 14 & 24 & 24 & 0 & 10 & 19 & 8 \\
\hline 5 & 18 & 19 & 29 & 20 & 22 & 26 & 23 & 0 & 16 & 22 & 11 \\
\hline 6 & 17 & 18 & 23 & 19 & 10 & 27 & 20 & 0 & 17 & 21 & 8 \\
\hline 7 & 16 & 19 & 25 & 18 & 25 & 23 & 26 & 0 & 19 & 20 & 20 \\
\hline 8 & 20 & 20 & 24 & 19 & 9 & 27 & 19 & 0 & 18 & 25 & 8 \\
\hline 9 & 19 & 21 & 28 & 22 & 8 & 24 & 24 & 0 & 18 & 22 & 7 \\
\hline 10 & 21 & 21 & 22 & 20 & 22 & 27 & 25 & 0 & 16 & 25 & 23 \\
\hline 11 & 16 & 17 & 26 & 21 & 22 & 27 & 23 & 0 & 16 & 19 & 10 \\
\hline 12 & 20 & 22 & 27 & 22 & 13 & 25 & 26 & 0 & 20 & 23 & 19 \\
\hline 13 & 13 & 15 & 24 & 16 & 22 & 24 & 23 & 0 & 11 & 19 & 21 \\
\hline 14 & 12 & 13 & 22 & 19 & 9 & 25 & 20 & 0 & 8 & 18 & 18 \\
\hline 15 & 19 & 21 & 27 & 21 & 7 & 24 & 19 & 0 & 18 & 22 & 0 \\
\hline 16 & 16 & 17 & 26 & 13 & 0 & 26 & 0 & 0 & 15 & 19 & 0 \\
\hline 17 & 18 & 21 & 23 & 19 & 10 & 25 & 9 & 0 & 19 & 22 & 9 \\
\hline 18 & 20 & 20 & 25 & 21 & 27 & 28 & 26 & 0 & 17 & 23 & 18 \\
\hline 19 & 19 & 22 & 21 & 22 & 22 & 26 & 23 & 19 & 20 & 23 & 20 \\
\hline 20 & 17 & 18 & 22 & 19 & 7 & 26 & 17 & 0 & 16 & 19 & 8 \\
\hline 21 & 18 & 18 & 23 & 18 & 12 & 27 & 25 & 0 & 17 & 20 & 9 \\
\hline 22 & 19 & 20 & 25 & 20 & 11 & 29 & 23 & 0 & 20 & 20 & 7 \\
\hline 23 & 20 & 21 & 22 & 21 & 13 & 27 & 26 & 0 & 20 & 22 & 19 \\
\hline 24 & 18 & 19 & 21 & 17 & 21 & 28 & 24 & 0 & 17 & 19 & 18 \\
\hline 25 & 20 & 20 & 22 & 20 & 12 & 30 & 23 & 0 & 19 & 21 & 9 \\
\hline 26 & 17 & 19 & 21 & 20 & 22 & 26 & 21 & 0 & 17 & 19 & 11 \\
\hline 27 & 11 & 12 & 12 & 0 & 8 & 25 & 8 & 0 & 0 & 17 & 7 \\
\hline 28 & 20 & 20 & 22 & 15 & 0 & 28 & 0 & 0 & 14 & 22 & 0 \\
\hline 29 & 19 & 21 & 23 & 19 & 24 & 26 & 23 & 20 & 17 & 24 & 19 \\
\hline 30 & 18 & 19 & 21 & 20 & 21 & 23 & 23 & 0 & 19 & 19 & 10 \\
\hline 31 & 19 & 19 & 24 & 20 & 12 & 26 & 24 & 0 & 18 & 23 & 8 \\
\hline 32 & 20 & 22 & 22 & 20 & 24 & 26 & 28 & 0 & 19 & 24 & 10 \\
\hline 33 & 20 & 21 & 21 & 19 & 22 & 24 & 24 & 0 & 18 & 23 & 11 \\
\hline 34 & 17 & 16 & 21 & 18 & 12 & 22 & 19 & 0 & 15 & 18 & 8 \\
\hline 35 & 10 & 9 & 10 & 0 & 0 & 23 & 0 & 0 & 8 & 14 & 0 \\
\hline 36 & 18 & 19 & 19 & 12 & 0 & 23 & 0 & 0 & 18 & 19 & 0 \\
\hline 37 & 0 & 0 & 11 & 0 & 0 & 22 & 0 & 0 & 0 & 11 & 0 \\
\hline 38 & 16 & 18 & 24 & 0 & 0 & 21 & 0 & 0 & 17 & 16 & 0 \\
\hline 39 & 21 & 22 & 21 & 16 & 10 & 26 & 9 & 0 & 18 & 22 & 9 \\
\hline Mean valu & $17.2 \pm 3.9$ & $18.23 \pm 4.15$ & $22.49 \pm 4.1$ & $17.1 \pm 6.2$ & $13.4 \pm 8.2$ & $25.64 \pm 2.13$ & $18.5 \pm 9.1$ & $1 \pm 4.4$ & $15.87 \pm 4.77$ & $20.64 \pm 3$ & $10.1 \pm 6.7$ \\
\hline
\end{tabular}

Legend: Amp: Ampicillin, CRO: Cefotriaxone, C: Chloramphenicol, CIP: Ciprofloxacin, CD: Clindamycin, E: Erythromycin, GN: Gentamycin, MTZ: Metronidazole TE: Tetracyclin, SXT: Sulfamethoxazole/trimethoprim

Table 2. Zones of inhibition diameters of kefir suspension $(0.1 \mathrm{~mL}$ culture grown for $24 \mathrm{~h}$ ), and the antimicrobial agents tested. Results showed the activity of kefir against $97.4 \%$ of the tested isolates. Ampicillin, chloramphenicol, ceftriaxone, erythromycin and gentamicin disks produced zone sizes generally similar to those 
of the kefir suspension. Kefir disks produced zone sizes higher than the zones produced by ciprofloxacin, sulphamethoxazole /trimethoprim and tetracycline disks. The mean values of the kefir inhibition zones were 17.2, standard deviations $3.9 \mathrm{~mm}$ and medians of $18 \mathrm{~mm}$.

\section{DISCUSSION}

Globally, BV causes genital problems among women that require gynecological care. BV has gained clinical importance since the condition has been associated with an increased susceptibility to HIV, herpes simplex viruses, sexually transmitted infections, and human papillomavirus (Cherpes et al., 2005; Cohen et al., 2012)

Several potential microbial pathogens have been involved in the BV development. Recent studies have reported that G. vaginalis can be a primary pathogen of BV disorder (Machado et al., 2013; Alves et al., 2014; Castro et al., 2019). The treatment and control of $B V$ can be employed by reducing the inhabitants of anaerobic bacteria, possibly prompting an increase in $\mathrm{H}_{2} \mathrm{O}_{2}$ lactobacilli-producing species (Giraldo et al., 2007). The antimicrobials recommended for BV are metronidazole and clindamycin (CDC, 2015). The efficacy of the afore said treatments are not optimal, as a high degrees of relapse and bacterial antibiotic resistance is commonly reported. The current study evaluated the antimicrobial susceptibility patterns in $G$. vaginalis to antibiotics that are recommended in routine BV treatment empirically and regionally. $G$. vaginalis isolates were found to have resistance to tetracycline $(94.7 \%)$ and sulphamethoxazole/trimethoprim $(74.4 \%)$ and were $100 \%$ sensitive to clindamycin followed by metronidazole $(89.7 \%)$. Even with the availability of effective antibioticsfor the treatment of BV, recurrence occurs within a year in approximately $60 \%$ of the BV cases (Bradshaw et al., 2006). Moreover, there was great concern regarding antimicrobial treatment as it is related to the suppression of vaginal microbiome, which is essential for homeostasis of the vaginal microbiota (Gajer et al., 2012). Besides, the recommended therapy, metronidazole and clindamycin, are associated with side effects, including gastrointestinal effects (Brzezinski et al., 2018). Consequently, novel therapeutic approaches are required for more success in the treatment of BV. The use of probiotics has received attention as a means of a treatment and preventve option for vaginal disorders. Kefir is the one of the probiotics claimed to have clinical effects on urinary tract infections, diarrheal diseases, Helicobacter pylori, streptococcal, and salmonella infections (Bourrie et al., 2016). Moreover, kefir grains have been used successfully to treat vaginal infections, due to its ability to produce antimicrobial compounds (Bourrie et al., 2016, Brzezinski et al., 2018). Although antimicrobial activity of kefir against many pathogen has been reported (Bourrie et al., 2016), antimicrobial activity of the kefir grains against $G$. vaginalis have not been previously described. This work has shown kefir activity against $G$. vaginalis isolates from BV patients. The kefir displayed activity against $97.4 \%$ of $G$. vaginalis isolates with zones of inhibition $\geq 10 \mathrm{~mm}$. Inhibition zones of kefir suspension in this study were of similar size as those reported against S. aureus, E.coli, Salmonella species, shigella species and bacillus species (Garrote et al., 2000; Mohammed \& Twaina, 2017). On the other hand, the study by Guzel-Seydim et al., (Guzel-Seydim et al., 2016), evaluated the antimicrobial activity of kefir against Fusobacterium nucleatum, they reported that the maximum inhibition zones was $9.5 \mathrm{~mm}$.

The plausible effects of the Kefir could be from the production of carbon dioxide, hydrogen peroxyde, bactericides, organic acids and ethanol during fermentation process. Additionally, the antimicrobial activity of kefir are owed to lactic acid and antibiotics produced by bacteria and yeasts (Bourrie et al., 2016).

\section{CONCLUSION}

The data presented in this work suggest that kefir may be good antimicrobial agent for use in $G$. vaginalis infections which is a leading cause of BV. Therefore, kefir could be a promising alternative for protection against $G$. vaginalis in women, thus preventing BV. However, further investigation with in vivo studies is required.

Acknowledgement: Authors acknowledge Dr. Ayat I. Younes, MD., Gynecology Clinic, El-Monib Family Medicine Center, Giza, Egypt, for providing clinical specimens.

\section{REFERENCES}

Alves, P., Castro, J., Sousa, C., Cereija, T.B., \& Cerca, N. (2014). Gardnerella vaginalis outcompetes 29 other bacterial species isolated from patients with bacterial vaginosis, using in an in vitro biofilm formation model. J. Infect. Dis., 210, 593-6. https://doi.org/10.1093/infdis/jiu131

Bostwick, D.G., Woody, J., Hunt, C., \& Budd, W. (2016). Antimicrobial resistance genes and modelling of treatment failure in bacterial vaginosis: clinical study of 289 symptomatic women. J. Med. Microbiol., 65, 377-386. https://doi.org/10.1099/jmm.0.000236

Bourrie, B.C.T., Willing, B.P., \& Cotter. P.D. (2016). The Microbiota and Health Promoting Characteristics of the Fermented Beverage Kefir. Front. Microbiol., 7, 647. https://doi.org/10.3389/fmicb.2016.00647

Bradshaw, C.S., Morton, A.N., Hocking, J., Garland, S.M., Morris, M.B., Moss, L., M., Horvath, L.B., Kuzevska, I., \& Fairley, C.K. (2006). High recurrence rates of bacterial vaginosis over the course of 12 months after oral metronidazole therapy and factors associated with recurrence. J. Infect. Dis., 193, 1478-1486. https://doi.org/10.1086/503780

Brzezinski, P., \& Martini, L., (2018). Kefir and champagne vinegar to defeat bacterial vaginosis in woman, avoiding oral metronidazole, clindamycin and bothersome douchings. Our Dermatol Online. 9(1), 22-24. DOI: 10.7241/ourd.20181.6.

Castro, J., Machado, D., \& Cerca, N. (2019). Unveiling the role of Gardnerella vaginalis in polymicrobial Bacterial Vaginosis biofilms: the impact of other vaginal pathogens living as neighbors. The ISME Journal. 13, 1306-1317. doi: 10.1038/s41396-018-0337-0.

Catlin, B.W., (1992). Gardnerella vaginalis: characteristics, clinical considerations, and controversies. Clinical Microbiology Reviews. 5, 213-237. DOI: 10.1128/CMR.5.3.213

CDC, 2015 https://www.cdc.gov/std/tg2015/bv.htm

Cherpes, T.L., Melan, M.A., Kant, J.A., Cosentino, L.A., Meyn, L.A., \& Hillier, S.L. (2005). Genital tract shedding of herpes simplex virus type 2 in women: effects of hormonal contraception, bacterial vaginosis, and vaginal group B Streptococcus colonization. Clinical Infectious Diseases. 40, 1422-1428. https://doi.org/10.1086/429622

CLSI. (2016). Performance Standards for Antimicrobial Susceptibility Testing. 26th ed. CLSI supplement M100S. Wayne, PA: Clinical and Laboratory Standards Institute; 2016. http://ljzx.cqrmhospital.com/upfiles/201601/20160112155335884.pdf

Cohen, R., Lingappa, J.R., Baeten, J.M., Ngayo, M.O., Spiegel, C.A., Hong, T., Donnell, D., Celum, C., Kapiga, S., Delany, S., \& Bukusi, E.A., (2012). Bacterial vaginosis associated with increased risk of female-to-male HIV-1 transmission: a prospective cohort analysis among African couples. PLoS Medicine. 9, e1001251. https://doi.org/10.1371/journal.pmed.1001251

Food and Agriculture Organization/World Health Organization. (2001). Health and nutritional properties of probiotics in food including powder milk with live lactic acid bacteria. Cordoba, Argentina: American Cordoba Park Hotel. Rome, Italy: FAO/WHO; 2001. p. 1-2.

Gajer, P., Brotman, R.M., Bai, G., Sakamoto, J., Schütte, U.M., Zhong, X, \& Ravel, J. (2012). Temporal dynamics of the human vaginal microbiota. Science translational medicine. 4(132), 132ra52. DOI: 10.1126/scitranslmed.3003605

Garrote, G.L., Abraham, A.G., \& De Antoni, G.L. (2000). Inhibitory Power of Kefir: The Role of Organic Acids. Journal of Food Protection. 63(3), 364-369. https://doi.org/10.4315/0362-028X-63.3.364

Giraldo, P.C., Passos, M.R.L., Bravo, R., Varella, R.Q., Campos, W.N.A., Amaral, R.L., \& Marussi, E. (2007). Challenge of understanding and dealing with bacterial vaginosis. DST - J. Bras. Doenças. Sex Transm., 19, 84-91.

Güzel-Seydim, Z.B., Cagdas, M.D.E., \& Seydim, A.C. (2016). Effect of kefir on Fusobacterium nucleatum in potentially preventing intestinal Cancer. Functional Foods in Health and Disease. 6 (7), 469-477. DOI: 10.31989/ffhd.v6i7.272

Machado, A., Jefferson, K.K., \& Cerca, N. (2013). Interactions between Lactobacillus crispatus and bacterial vaginosis (BV)-associated bacterial species in initial attachment and biofilm formation. Int. J. Mol. Sci., 14, 12004-12. https://doi.org/10.3390/ijms140612004

Mohammed, A.A. \& Twaina, A.A. (2017). An in Vitro Study on the Antibacterial Activity of Kefir Milk, Labzyme, Biozyme and Some Antibiotics against Staphylococcus aureus and Escherichia coli causes Lameness in Broiler Chickens. IJSER., 5 (7), $239-242$ 
Rodrigues, K.L., Caputo, L.R., Carvalho, J.C., Evangelista, J., \& Schneedorf, J.M., (2005). Antimicrobial and healing activity of kefir and kefir an extract. Int.

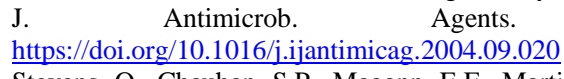

Stevens, O., Chauhan, S.P., Magann, E.F., Martin, R.W., Bofill, J.A., Cushman, J.L., \& Morrison, J.C. (2004). Fetal fibronectin and bacterial vaginosis are associated with preterm birth in women who are symptomatic for preterm labor. American Journal of Obstetrics \& Gynecology. 190, 1582-1587. https://doi.org/10.1016/j.ajog.2004.03.059 